\title{
Genetic Dissection of Cancer Development, Therapy Response, and Resistance in Mouse Models of Breast Cancer
}

\author{
Stefano Annunziato, ${ }^{1}$ Marco Barazas, ${ }^{1}$ Sven Rottenberg, ${ }^{1,2}$ and Jos Jonkers ${ }^{1,3}$ \\ ${ }^{1}$ Division of Molecular Pathology, The Netherlands Cancer Institute, 1066 CX Amsterdam, \\ The Netherlands \\ ${ }^{2}$ Institute of Animal Pathology, Vetsuisse Faculty, University of Bern, 3012 Bern, Switzerland \\ ${ }^{3}$ Cancer Genomics Netherlands, The Netherlands Cancer Institute, 1066 CX Amsterdam, \\ The Netherlands \\ Correspondence: j.jonkers@nki.nl; sven.rottenberg@vetsuisse.unibe.ch
}

\begin{abstract}
The cancer genomics revolution has rapidly expanded the inventory of somatic mutations characterizing human malignancies, highlighting a previously underappreciated extent of molecular variability between and within patients. Also in breast cancer, the most commonly diagnosed malignancy in women, this heterogeneity complicates the understanding of the stepwise sequence of pathogenic events and the design of effective and long-lasting target therapies. To disentangle this complexity and pinpoint which molecular perturbations are crucial to hijack the cellular machinery and lead to tumorigenesis and drug resistance, functional studies are needed in model systems that faithfully and comprehensively recapitulate all the salient aspects of their cognate human counterparts. Mouse models of breast cancer have been instrumental for the study of tumor initiation and drug response but also involve cost and time limitations that represent serious bottlenecks in translational research. To keep pace with the overwhelming amount of hypotheses that warrant in vivo testing, continuous refinement of current breast cancer models and implementation of new technologies is crucial. In this review, we summarize the current state of the art in modeling human breast cancer in mice, and we put forward our vision for future developments.
\end{abstract}

Breast cancer is the most commonly diagnosed invasive cancer worldwide, with more than 1.6 million new cases each year. Rather than a single disease, it represents a spectrum of malignancies, encompassing several distinct biological entities and subtypes, each associated with specific histopathological and molecular characteristics, responses to therapy, and clinical outcomes. Multiple taxonomies have been developed to divide breast cancer cases into different categories. Histopathological classification comprises several morphological and immunohistochemical phenotypes that can be further divided into different grades. Among the various morphologies, advanced mammary tumors mostly fall into the class of invasive ductal carcinomas (IDCs), followed by invasive lobular carcinomas (ILCs). Molecular classification based on gene expression patterns distinguishes five major subtypes of breast cancer: luminal A and B, ErbB2 ${ }^{+}$, basal-like, and claudin-low (Perou et al. 2000). Although these distinctions have proven useful for clinical decisionmaking, there are limitations in predicting disease prognosis and response to therapy. For example, a recent prospective, randomized phase III study showed that nearly half of the women with early breast cancer who are at high risk based on standard clinicopathological parameters might not require adjuvant chemotherapy (Cardoso et al. 2016). The additional use of a 70-gene expression signa- ture may help to identify breast cancer patients who do not require adjuvant chemotherapy, but the identification of molecular signatures that reliably predict chemotherapy response remains elusive. Moreover, sequencing studies have shown that even within the same molecular subtype an extreme heterogeneity in the mutational landscape exists, which may account for discrepancies in prognosis and therapy response between different patients (Cancer Genome Atlas Network 2012; Stephens et al. 2012; NikZainal et al. 2016). Another complicating factor is intratumoral heterogeneity. Individual tumors are mosaics of multiple clones of neoplastic cells, each characterized by a distinct genetic makeup and differential responses to the selective pressures to which they are exposed, making the tumor mass not static but continuously shaped by a branching evolutionary process resembling Darwinian evolution. Distinguishing causal disease variants (driver mutations) from background alterations (passenger mutations) is a major goal in breast cancer research, as it can pinpoint evolutionary conserved processes that mammary tumor cells apply during stepwise transformation and to which they might be addicted. To exploit these potential Achilles' heels, we require a comprehensive knowledge of how these signaling networks physiologically function, how they become aberrant, and how they can be directly or indirectly disrupted.

\footnotetext{
(C) 2016 Annunziato et al. This article is distributed under the terms of the Creative Commons Attribution-NonCommercial License, which permits reuse and redistribution, except for commercial purposes, provided that the original author and source are credited.
} 
Given this complexity, genetically engineered mouse models (GEMMs) of breast cancer, together with patientderived tumor xenografts (PDXs) and GEMM-derived tumor allografts, have proven valuable resources for deepening our understanding of how mammary tumors initiate, progress, metastasize, and respond to therapy in a physiologically relevant in vivo setting (Vargo-Gogola and Rosen 2007). These mouse models are increasingly being used in longitudinal preclinical studies for translation of novel therapies to clinical testing. Moreover, GEMMs provide unique opportunities to infer causeeffect relationships on de novo induced malignancies growing in intact organisms, rather than correlative observations on end-stage patient tumor samples.

Over the past 15 years, our research has been focused on the generation and characterization of mouse models for two breast cancer subtypes: ILCs and basal-like IDCs. To achieve this, we engineered a number of tumor-specific driver mutations in the relevant target cells of mouse models, recapitulating the key dependencies of the resulting lesions to the corresponding deranged signaling pathways. In this review, we will discuss how these models can be used for functional dissection of tumorigenic cascades, unraveling new therapeutic vulnerabilities and mechanisms of therapy resistance - in particular, in light of the advent of new technologies such as clustered regularly interspersed short palindromic repeat (CRISPR)Cas9 gene editing, which are opening new avenues in breast cancer modeling in mice.

\section{INVASIVE LOBULAR BREAST CARCINOMA (ILC) MODELS}

ILC accounts for $8 \%-14 \%$ of all breast cancer cases and is hallmarked at the morphological level by tumor cells growing in single "Indian files" within a dense fibrous stroma. This phenotype can be explained at the molecular level by loss of integrity of cell adherens junctions because of mutations or methylation of the $\mathrm{CDH1}$ gene, which encodes the transmembrane protein E-cadherin (Martinez and Azzopardi 1979; Borst and Ingold 1993; Moll et al. 1993; Vos et al. 1997; Droufakou et al. 2001). To our surprise, we found that mammary glandspecific Cre-mediated inactivation of $C d h 1$ alleles in mice was insufficient to induce mammary tumors, probably because normal cells undergo apoptosis and are counterselected when E-cadherin is lost (Boussadia et al. 2002; Derksen et al. 2006, 2011). This prompted us to investigate which cooperating oncogenic events are required for malignant transformation of E-cadherin-deficient mammary epithelial cells. We have found that multifocal ILC formation is promoted by dual mammary-specific loss of E-cadherin and p53 (Derksen et al. 2006, 2011) or E-cadherin and PTEN (phosphatase and tensin homolog) (Boelens et al. 2016), with tumor architecture and molecular profiles closely resembling their human ILC counterparts (Table 1). However, it remains elusive which biological processes are rescued by codepletion of E-cadherin with one of these factors. To identify
Table 1. Characteristics of human ILC and BRCA1-associated breast cancer and the corresponding GEMMs developed in our laboratory

\begin{tabular}{llc}
\hline $\begin{array}{l}\text { Invasive lobular } \\
\text { carcinoma }\end{array}$ & Human & $\begin{array}{c}\text { WAPcre; } \text { CdhI }^{F / F} ; \text { Pten }^{F / F} \\
\text { mouse model }\end{array}$ \\
\hline Morphology & Lobular & Lobular \\
Invasive & Yes & Yes \\
Grade & Low & Low \\
Mitotic index & Low & Low \\
ER expression & Yes & Yes \\
Molecular subtype & Luminal & Luminal \\
Collagen deposition & Yes & Yes \\
Stroma-rich & Yes & Yes \\
\hline BRCA1-associated & & K14cre; Brcal ${ }^{F / F} ; p 53^{F / F}$ \\
breast cancer & Human & mouse model \\
\hline Morphology & Ductal & Ductal \\
Invasive & Yes & Yes \\
Grade & High & High \\
Mitotic index & High & High \\
ER/PR expression & No & No \\
Molecular subtype & Basal-like & Basal-like \\
Genomically & Yes & Yes \\
instable & Yes & Yes \\
HR-deficient & Pes
\end{tabular}

ILC, invasive lobular carcinoma; BRCA1, breast cancer gene 1; GEMMs, genetically engineered mouse models; ER, estrogen receptor; $\mathrm{PR}$, progesterone receptor; HR, homologous recombination.

novel candidate cancer genes and networks that collaborate with E-cadherin loss in mammary tumorigenesis, we used the Sleeping Beauty (SB) transposon system (Collier et al. 2005; Dupuy et al. 2005) to perform an insertional mutagenesis screen in WAPcre; $C d h 1^{F / F}$ mice (SM Kas, $\mathrm{J}$ de Ruiter, K Schipper, et al., in prep.). Retrieval of recurrent integrations in SB-induced WAPcre;Cdh1 $1^{F / F}$ mammary tumors identified common insertion sites in several genes, some known to be mutated in human ILC, suggesting that mutagenesis of these genes leads to malignant transformation. Moreover, analysis of enriched targeted pathways and mutually exclusive insertions revealed the regulation of the actin cytoskeleton as a completely novel oncogenic pathway in both mouse and human ILC.

Furthermore, recent genomic studies on collections of human ILCs have unveiled that, in addition to somatic inactivation of E-cadherin, activation of phosphoinositide 3-kinase (PI3K)/AKT signaling appears to be a common event in this breast cancer subtype (Ciriello et al. 2015; Desmedt et al. 2016; Michaut et al. 2016). To validate these findings, we developed GEMMs of ILC that combine mammary gland-specific ablation of E-cadherin and activation of different oncogenic Pik3ca or Akt mutants (MHAM van Miltenburg, et al., in prep.). To rapidly generate breast cancer models carrying these allelic variants, we used a novel strategy for fast-track production of GEMMs, called GEMM-ESC, which is based on Flprecombinase-mediated introduction of additional mutant alleles into the Collal locus of embryonic stem cells (ESCs) derived from existing GEMMs (Huijbers et al. 2014). Interestingly, the resulting mice showed rapid development of tumors with strong resemblance to human ILC in terms of morphology, gene expression, and invasiveness, on which we are now testing a panel of antican- 
cer therapeutics to identify promising genotype-specific drug sensitivities.

\section{BASAL-LIKE BREAST CANCER MODELS}

Basal-like breast cancers represent a heterogeneous class of malignancies with poor clinical outcome that accounts in total for $10 \%-15 \%$ of all breast cancer cases (Perou et al. 2000; Badve et al. 2011). The majority of basal-like tumors lack expression of estrogen receptor (ER), progesterone receptor (PR), and human epidermal growth factor receptor 2 (HER2) and are therefore referred to as triple-negative breast cancers. These tumors are not targetable with hormonal therapy or HER2 inhibitors, which leaves clinicians with only few effective options for therapeutic intervention.

Approximately $50 \%$ of basal-like breast cancers display a dysfunctional BRCA pathway because of germline or somatic mutations in BRCA1/2 or BRCA1 promoter hypermethylation (Cancer Genome Atlas Network 2012; Nik-Zainal et al. 2016). Also a fraction of non-basal-like tumors are BRCA-deficient, mostly because of germline mutations in BRCA2. As these genes are crucial in the error-free repair of DNA double-strand breaks (DSBs) by homologous recombination (HR), BRCA defects are associated with chromosomal instability and hypersensitivity to DNA DSB-inducing drugs such as alkylating agents, poly ADP ribose polymerase inhibitors (PARPi), and radiotherapy (Jaspers et al. 2009; Bouwman and Jonkers 2012; M Barazas, et al., in prep.). However, drug resistance mechanisms have been described in both clinical and preclinical studies of BRCA-associated tumors, posing serious concerns, as no other therapies are currently available for relapsing patients.

To study tumorigenesis and drug resistance mechanisms, we developed several conditional mouse models for BRCA1- and BRCA2-associated breast cancer (Evers and Jonkers 2006; Bouwman and Jonkers 2008). In our K14cre;Brcal ${ }^{F / F} ; p 53^{F / F}$ (KB1P) and K14cre;Brca2 $2^{F / F}$; $p 53^{F / F}$ (KB2P) models, mammary inactivation of Brcal/ 2 is accompanied by loss of p53, as mutations in this tumor suppressor frequently co-occur with $B R C A 1 / 2$ mutations in breast cancer (Jonkers et al. 2001; Liu et al. 2007). These mice develop mammary tumors after a latency period of 6-8 mo, suggesting that additional mutations are required for tumorigenesis (Table 1). However, in contrast to ILC, in which point mutations are the most common somatic alterations, BRCA-mutated breast cancers are characterized by complex patterns of DNA copy number aberrations (CNAs), including translocations and gains/losses of entire chromosome arms (Vollebergh et al. 2012). Using cross-species oncogenomics, we identified $M Y C$ amplification and $R B 1$ loss as recurrent CNAs in both mouse and human BRCA1/2-mutated breast cancers (Holstege et al. 2010). Exploiting the GEMM-ESC strategy, we could model conditional overexpression of MYC in our WAPcre;Brcal ${ }^{F / F} ; p 53^{F / F}$ (WB1P) mouse model and found that mammary tumor development was indeed strongly accelerated compared to the original line (L Henneman, et al., in prep.). Moreover, we observed that the number of CNAs in WB1PMYC tumors was markedly reduced compared to WB1P tumors, showing only few recurrent CNAs that most likely harbor additional cancer drivers that collaborate with MYC overexpression and loss of BRCA1/p53 in breast tumorigenesis. We are currently performing cross-species comparisons of the recurrent CNAs in WB1PMYC tumors with CNA profiles from human breast cancers to identify candidate cancer genes, which will be validated in the WB1P-MYC model. We believe that this iterative CNA profiling approach in progressively complex GEMMs will be instrumental for deciphering the key driver events in BRCA1-associated breast cancer and for uncovering novel therapeutic vulnerabilities.

\section{PRECLINICAL TRIALS IN BRCA- ASSOCIATED BREAST CANCER MODELS}

Although phase-I and -II clinical trials are mostly carried out in heavily pretreated volunteer patients who suffer from end-stage metastatic cancer, mouse models provide the opportunity to initiate treatment on naïve tumors in a clinically relevant in vivo setting. Treatment of mammary tumor-bearing KB1P mice with a panel of DSB-inducing agents showed heterogeneous responses between individual tumors but also marked differences in tumors treated with doxorubicin or docetaxel and those treated with cisplatin (Rottenberg et al. 2007). Although KB1P tumors eventually developed resistance to doxorubicin and docetaxel, no acquired resistance was observed for cisplatin. Even though these tumors could never be completely eradicated by maximum tolerated dose concentrations of cisplatin, the relapsing tumors remained responsive to subsequent treatments, resulting in a typical sawtooth tumor response. A major breakthrough came when it was found that spontaneous KB1P and KB2P tumors could be orthotopically allografted in syngeneic mice while maintaining their genetic characteristics and drug sensitivity profile. This approach reduced the time to produce cohorts of tumor-bearing mice from 7-9 mo to 4-6 wk, and enabled large-scale intervention studies in which the response of a single donor to different chemotherapeutic strategies could be compared, ruling out any intertumor heterogeneity (Fig. 1). Intervention studies with the PARP inhibitor olaparib in KB1P tumor allografts led to the development of carboplatin and olaparib switch-maintenance therapy for BRCA1-mutated breast cancer (Rottenberg et al. 2008). This preclinical concept was confirmed in a clinical trial with olaparib maintenance therapy in BRCA-mutation carriers with platinum-sensitive ovarian cancer (Ledermann et al. 2012, 2014) and eventually led to clinical approval of olaparib (Deeks 2015). Similarly, intervention studies in KB2P tumor allografts showed that alkylators such as nimustine could induce complete tumor eradication (Evers et al. 2010). Eradication of $B R C A$-mutated and BRCA-like cancer by high-dose alkylating chemotherapy was subsequently confirmed by 


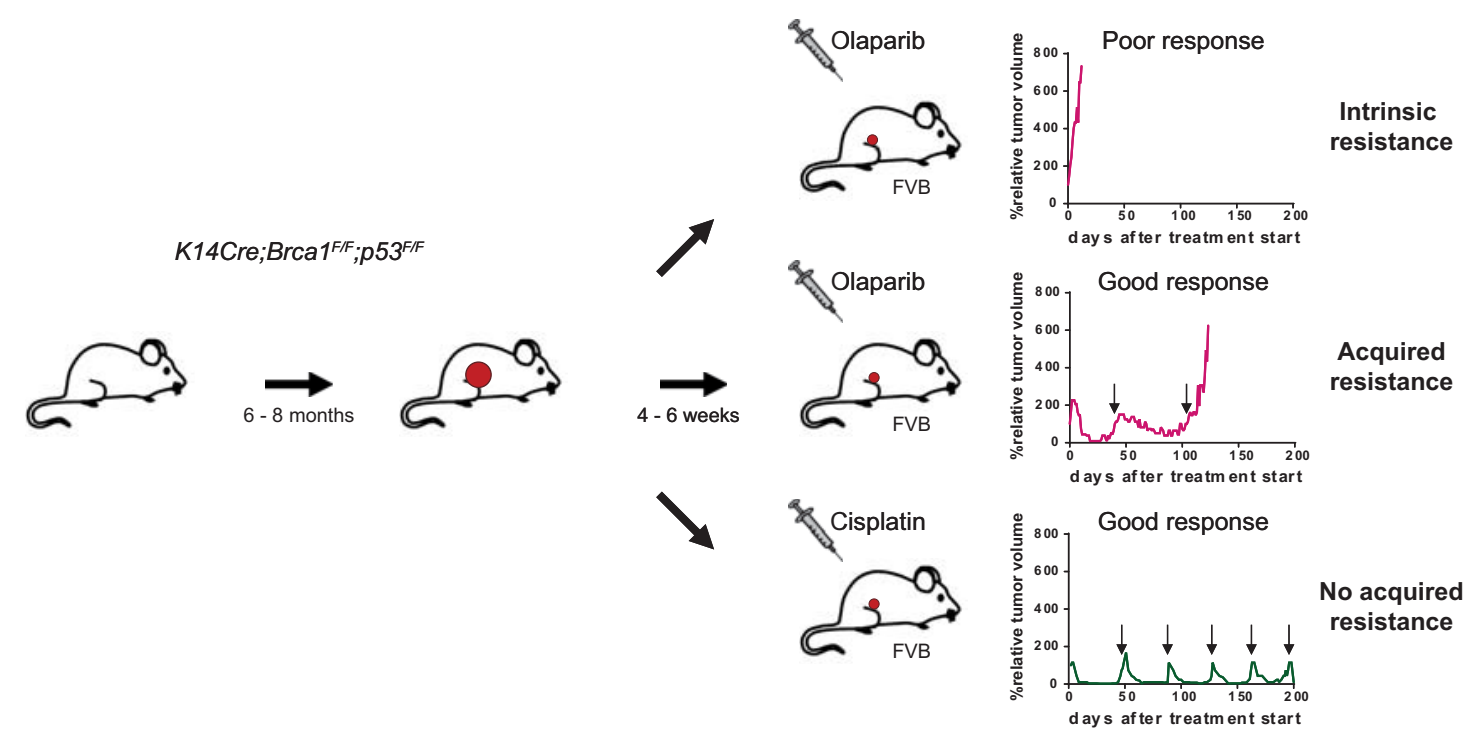

Figure 1. Large-scale intervention studies using breast cancer gene (BRCA)-deficient orthotopic allografts. Spontaneous tumors develop with a latency of $6-8$ mo in K14cre;Brcal ${ }^{F / F} ; p 53^{F / F}(\mathrm{~KB} 1 \mathrm{P})$ or K14cre;Brca2 $2^{F / F} ; p 53^{F / F}$ (KB2P) genetically engineered mouse models (GEMMs). Retransplantation of spontaneous tumors in syngeneic FVB wild-type mice highlighted intertumor heterogeneity in treatment response. In the case of olaparib, some tumors displayed intrinsic resistance, whereas others displayed initial good response followed by the emergence of acquired resistance. In contrast, resistance never developed in cisplatin-treated animals, despite multiple consecutive rounds of treatment (indicated by black arrows).

retrospective analysis of data from clinical trials (Vollebergh et al. 2011, 2014; Schouten et al. 2015). These and other studies illustrate the utility of GEMMs of human cancer in translational cancer medicine.

\section{PARPi RESISTANCE MECHANISMS IN BRCA- ASSOCIATED BREAST CANCER MODELS}

In addition to accelerating preclinical trials, the KB1P and KB2P allograft platforms also enabled large-scale induction of acquired resistance to a drug of choice and subsequent identification of the underlying resistance mechanisms. The power of this approach was demonstrated with the PARP inhibitor olaparib, which was described to display selective toxicity against BRCA1/2-deficient cells (Bryant et al. 2005; Farmer et al. 2005). Indeed, KB1P tumor allografts initially responded well to treatment but eventually relapsed and developed stable resistance (Rottenberg et al. 2008). This has provided a valuable collection of matched treatment-naïve and treatment-resistant tumors, which could be analyzed using next-generation sequencing or (phospho)-proteomics, thereby taking advantage of the clean genetic background of inbred mice and the known genetic profile of treatment-naïve tumors. We found that $A b c b l a$ and $A b c b 1 b$, encoding P-glycoprotein (P-gp) efflux pumps, were upregulated in resistant tumors and we confirmed that P-gp played an important role in mediating export of olaparib from tumor cells (Fig. 2A; Rottenberg et al. 2008). Resistance could be reversed when P-gp-mediated drug efflux was inhibited by coadministration of tariquidar. Although the clinical relevance of P-gp up-regulation as cause of drug resistance remains controversial (Amiri-
Kordestani et al. 2012), expression of $M D R 1$, the human counterpart of $A b c b 1$, was recently found to be inversely correlated to olaparib response in human ovarian cancer cells (Vaidyanathan et al. 2016). Such increased expression may result from complex genomic rearrangements that fuse a distant promoter to the MDR1 gene and thereby bypass the MDR1 promoter methylation (Patch et al. 2015). The case of P-gp shows that a thorough mechanistic understanding is instrumental to combat resistant tumors - for example, by coadministration of tariquidar or by switching treatment to chemotherapeutics that are poor substrates for P-gp (Jaspers et al. 2013).

To dissect P-gp-independent mechanisms of PARPi resistance, the KB1P mouse model was refined through germline genetic deletion of $M d r l$ resulting in the K14cre; Brcal ${ }^{F / F} ;{\text { p } 53^{F / F} ; M d r l a / b^{-/-}}(\mathrm{KB} 1 \mathrm{PM})$ model (Jaspers et al. 2013). Alternatively, KB1P tumors were treated with the PARP inhibitor AZD2461, which is a poor substrate for P-gp (Oplustil O'Connor et al. 2016). PARPi resistance developed in these models despite the exclusion of P-gp-related mechanisms. To identify the underlying resistance mechanisms, next-generation sequencing data from treatment-naïve and PARPi-resistant tumors were combined with data from unbiased functional genetic screens in vitro. Through an insertional mutagenesis screen in conditional BRCA1-knockout mouse ESCs, we found that loss of 53BP1 rescues the proliferation defect, HR deficiency, and PARPi hypersensitivity of BRCA1-deficient cells by enhancing DSB end resection (Fig. 2B; Bouwman et al. 2010). This work from our laboratory and similar studies from the Nussenzweig laboratory (Bunting et al. 2010) have led to novel mechanistic insights in DSB repair and to date several downstream effector proteins of 53BP1 have been shown to suppress 
A
Up-regulation of drug efflux pump

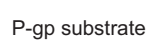

(i.e., olaparib)

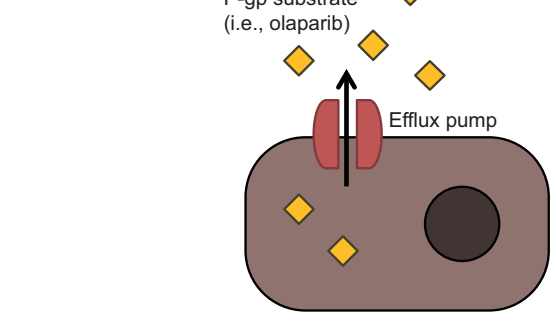

B

Rewiring DNA damage response

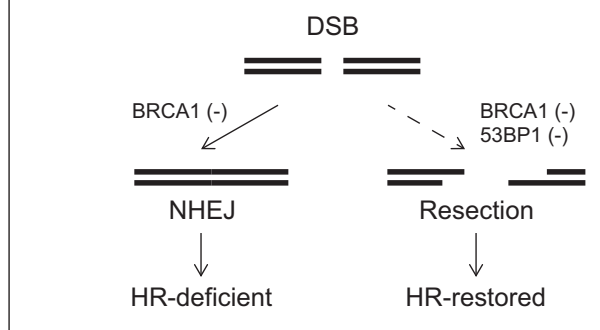

\section{C}

\section{Genetic reactivation}

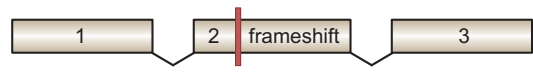

1. Alternative splicing

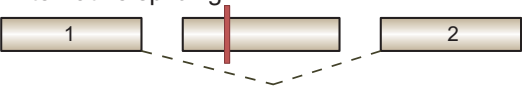

2. Retromutation

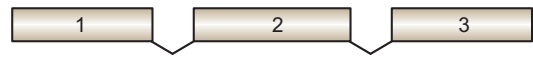

3. Secondary mutation

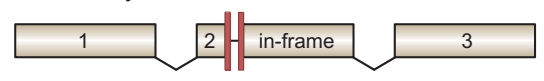

D Transcriptional reactivation

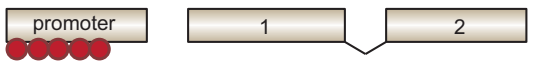

1. Promoter demethylation

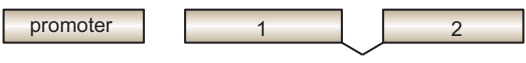

2. Gene fusion to heterologous promoter

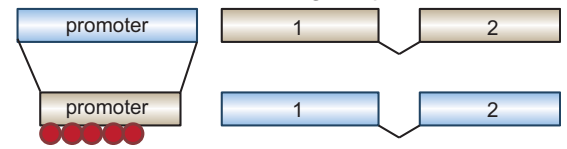

Figure 2. Overview of resistance mechanisms identified using mouse models of breast cancer gene (BRCA)-deficient breast cancer. $(A)$ Up-regulation of drug efflux pumps (i.e., MDR1) reduces intracellular drug concentration. (B) The DNA damage response pathway can be rewired to restore homologous recombination (HR), in this case by loss of 53BP1. (C) Genetic reactivation of BRCA1-mutated alleles can occur because of alternative splicing, retromutations, or secondary mutations restoring the $B R C A 1$ reading frame. $(D)$ Transcription of silenced BRCA1 alleles can be restored upon promoter demethylation or gene fusions to distant promoters. P-gp, Pglycoprotein; DSB, double-strand break; NHEJ, nonhomologous end joining; HR, homologous recombination.

HR in BRCA1-deficient cells, including RIF1 (Chapman et al. 2013; Di Virgilio et al. 2013; Escribano-Diaz et al. 2013; Zimmermann et al. 2013), PTIP (Callen et al. 2013), Artemis (Wang et al. 2014), and REV7/MAD2L2 (Boersma et al. 2015; Xu et al. 2015). Thorough analysis of mutational status and expression levels of 53BP1 and REV7 in PARPi-resistant KB1P(M) tumors confirmed that loss of 53BP1 or REV7 causes in vivo resistance to PARPi (Jaspers et al. 2013; Xu et al. 2015). Interestingly, although KB1P(M) tumors with 53BP1 loss are crossresistant to topotecan and doxorubicin, they are still responsive to cisplatin, suggesting that platinum drugs may be a useful salvage therapy for this class of PARPi-resistant tumors (Jaspers et al. 2013).

Although the majority of $\mathrm{KB} 1 \mathrm{P}(\mathrm{M})$ tumors acquired PARPi resistance through restoration of $\mathrm{HR}$, a substantial fraction of PARPi-resistant tumors remained defective in the formation of ionizing radiation-induced nuclear RAD51 foci (RAD51-IRIFs), which are a hallmark of HR. Moreover, when we analyzed the BRCA2-deficient KB2P tumors with acquired PARPi resistance, none of these showed restoration of HR as measured by RAD51IRIF assays (E Gogola, et al., in prep.). This suggests the existence of alternative resistance mechanisms. It was recently shown that chemoresistance in BRCA2-deficient cells might be mediated through protection of replication forks (RFs) - for instance, by depletion of PAX transcription activation domain interacting protein (PTIP) (Chaud- huri et al. 2016). It will be important to investigate whether RF protection is a common feature of PARPiresistant $\mathrm{KB} 1 \mathrm{P}(\mathrm{M})$ and $\mathrm{KB} 2 \mathrm{P}$ tumors.

In patients, mutated $\mathrm{BRCA} 1$ or BRCA2 proteins are often still expressed in tumors. Therefore, the large intragenic Brcal/2 deletions present in KB1P(M) and KB2P tumors - although instrumental in genetic studiesmight not fully recapitulate the biology of BRCA-associated tumors in mutation carriers. To this end, we generated several mouse models mimicking pathogenic $B R C A 1$ variants that are often encountered in the clinic (Drost et al. 2011, 2016). These models provided evidence that the type and location of the BRCA1 mutation can have significant implications for the response of these tumors to DSB-inducing agents and PARPi. It was found that tumor cells harboring the $B R C A 1^{185 d e l A G}$ allelic variant, which was modeled in mice by a Brcal $1^{185 \text { stop }}$ allele, can use a downstream alternative start site leading to the expression of a RING-less BRCA1 protein (Drost et al. 2016). This RING-less BRCA1 protein maintains hypomorphic HR activity, which is sufficient to induce a poor response to platinum drugs or olaparib. These results illustrate the importance of testing $B R C A 1$ allelic variants not only for genetic counseling but also for providing adequate treatment.

PDX models provide a solution to narrow the gap between mouse and human cancer biology and as such represent a novel in vivo platform for studying therapy 
response and resistance. Although PDX models have been relatively difficult to generate in the past, recent advances have made it possible to generate PDX biobanks covering a heterogeneous population of tumors (Hidalgo et al. 2014). Once the (epi)genetic landscape of a PDX model is characterized, this provides an effective tool to study the drug response of a specific tumor and to predict which resistance mechanisms might evolve during treatment. We recently demonstrated the feasibility of such an approach by treatment of PDX models of BRCA1-deficient breast cancer with alkylating agents or olaparib (Ter Brugge et al. 2016). Similar to the GEMM tumors, these PDX tumors generally responded well to treatment, but eventually developed resistance. The underlying mechanism was dependent on the type of $B R C A 1$ inactivation: Whereas therapy-resistant $B R C A 1$ methylated PDX tumors frequently showed $B R C A 1$ promoter demethylation, $B R C A 1$-mutated tumors acquired resistance via genetic reversion through secondary mutations that restored the $B R C A 1$ reading frame (Fig. 2C,D). These events have also been known to mediate resistance in ovarian cancer patients (Swisher et al. 2008; Patch et al. 2015), showing the predictive potential of PDX models. The PDX models also revealed a novel resistance mechanism involving gene fusions that placed BRCA1 under transcriptional control of a heterologous promoter. It is intriguing that resistance mechanisms in PDX tumors are mainly centered on re-expression of functional BRCA1 protein rather than inactivation of $53 \mathrm{BP} 1$ or related factors, highlighting the strong selective pressure on complete restoration of BRCA1 function when BRCA1deficient tumor cells are exposed to DSB-inducing therapy. However, a fraction of tumors acquired resistance in the absence of BRCA1 reexpression, showing that alternative resistance mechanisms also occur in PDX models.

Taken together, these studies illustrate the power of mouse models in unraveling resistance mechanisms before their emergence in patients. It will be important to investigate to what extent these play a role in the clinic. This is not trivial, as they likely occur in a limited group of $B R C A$ patients and thus require careful patient selection. It is noteworthy that resistance caused by mutations in additional DNA repair genes such as 53BP1 or REV7 might expose new treatment vulnerabilities (e.g., sensitivity to combined PARP and ataxia telangiectasia mutated [ATM] inhibition [Bunting et al. 2010]). It will therefore be important to determine if and how each resistance mechanism can be exploited therapeutically. U1timately, this may provide a framework for oncologists to combat resistance in the clinic.

\section{NONGERMLINE GEMMS OF BREAST CANCER}

Large-scale cancer genome sequencing studies and forward genetic screens have jointly boosted the discrimination between passenger and driver mutations and the identification of genetic determinants of drug sensitivity and resistance in breast cancer. The systematic translation of these long catalogs of structural aberrations into functional information requires the assessment of the pathophysiological impact of candidate gene perturbations in reliable preclinical models. This inevitably poses a practical challenge for in vivo validation experiments, because of the considerable costs and time requirements associated with establishing new breast cancer GEMMs. Novel technologies, especially CRISPR-Cas9-based methods, are revolutionizing the genetic engineering field by providing fast ways for precise and efficient ESC manipulation and GEMM development (Wang et al. 2013). However, as sequencing expenses of human tumors keep decreasing, research will shift from testing oncogenicity of single driver alleles to investigating the impact of multiple allelic variants on tumor development and therapy response. At the same time, forward genetics strategies will evolve from genome-wide approaches based on simple gene (in)activation to more refined chemical mutagenesis and gene-based CRISPR screens capable of identifying novel hypomorphic, dominant-negative, and separation-offunction mutants at the base pair level. We foresee that the number of testable hypotheses will far exceed the capacity of transgenic facilities, warranting the development of new in vivo platforms for systematic, multiplexed interrogation of putative cancer drivers. Ideally, such models should sort out current temporal and economical limitations of GEMM establishment and bypass extensive mouse husbandry but also allow a high degree of manipulability and flexibility by enabling spatiotemporal control of tumor initiation and progression.

To develop such a platform for breast cancer, we explored the possibility of nongermline modeling of mammary tumors by exploiting intraductal injection in the nipple of adult female mice as a way to deliver high-titer lentiviral or adenoviral preparations to mammary epithelium and achieve somatic genome engineering. We have shown that intraductally injected lentiviruses can target tumor-initiating cells of both the basal and the luminal compartment, allowing modeling of both ILC and basallike tumors in mice with the corresponding set of relevant predisposing alleles. For example, intraductal injection of Cre-encoding lentiviruses in $C d h 1^{F / F} ;$ Pten $^{F / F}$ mice induced the formation of ILCs that were undistinguishable from the ILCs arising in the original WAPcre; $C d h 1^{F / F}$; $P_{t e n}{ }^{F / F}$ model (Annunziato et al. 2016). Somatic Cre delivery may more accurately recapitulate sporadic tumor initiation by allowing titratable and spatiotemporally controlled delivery of viruses to mammary tissue. Moreover, targeting specificity can be modulated by using viruses with cell type-specific promoters and/or posttranscriptional control elements (Tao et al. 2014).

Importantly, the potential of nongermline modeling extends far beyond simple exogenous administration of Cre to established GEMMs. A diverse array of viral and nonviral constructs can be employed to achieve desired permutations of specific candidate genes even in the absence of germline conditional alleles: (a) vectors for overexpression of wild-type, truncated, or mutated cDNAs; (b) vectors for shRNA-mediated down-regulation or CRISPRmediated (epi)genetic manipulation of single or multiple 


\begin{tabular}{|c|c|c|}
\hline & $\begin{array}{l}\text { Somatic Cre delivery } \\
\text { for ILC modeling }\end{array}$ & \\
\hline $\begin{array}{c}\text { CRISPR manipulation } \\
\text { of a gene }\end{array}$ & (e.g., to $E c a d^{F / F} ; P^{2 t e n}{ }^{F / F}$ ) & $\begin{array}{l}\text { Somatic Cre delivery } \\
\text { for TNBC modeling }\end{array}$ \\
\hline (e.g., sgPten) & & (e.g., to $B r c a 1^{F / F} ; p 53^{F / F}$ ) \\
\hline
\end{tabular}

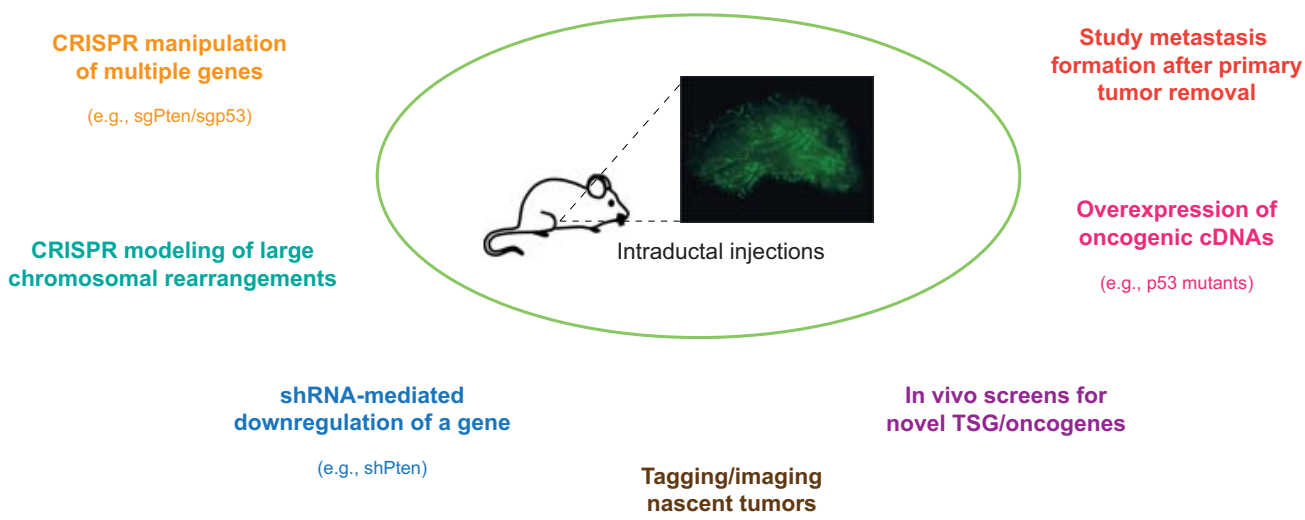

Figure 3. Multiple applications for somatic genome engineering of the mammary tissue via intraductal injection to study tumor biology. ILC, invasive lobular carcinoma; TNBC, triple-negative breast cancer; sgPten, single-guide phosphatase and tensin homolog; shPTEN, short-hairpin phosphatase and tensin homolog; TSG, tumor suppressor gene.

endogenous genes (Sander and Joung 2014); (c) CRISPR vectors for modeling large chromosomal rearrangements (Maddalo et al. 2014); and (d) vectors for tagging and imaging of tumors (Fig. 3). Regarding CRISPR-based in vivo editing approaches, we and others have shown that somatic delivery of the bacterial Cas9 protein has the considerable drawback of eliciting strong and specific immune responses in immunocompetent animals (Wang et al. 2015; Annunziato et al. 2016). This problem can be overcome by employing knock-in models that are tolerant to Cas9 because of constitutive or conditional expression of Cas 9 or catalytically inactive dCas9-effector fusions (which allow for transcriptional silencing/activation of endogenous alleles) (Platt et al. 2014; SánchezRivera and Jacks 2015). We have recently reported somatic induction of oncogenic loss-of-function mutations in mice with mammary-specific expression of Cas9 by intraductal injection of single-guide RNA (sgRNA)-encoding lentiviruses, which eventually led to ILC formation (Annunziato et al. 2016).

\section{MAMMARY TUMOR ORGANOIDS}

Another exciting technological breakthrough came from the possibility to derive organotypic 3D culture mod- els of normal and malignant mammary tissue. Human and murine tumor organoid cultures retain key features of donor tumors, including cellular heterogeneity and molecular characteristics (Clevers 2016; Fatehullah et al. 2016). Compared to the laborious and time-consuming establishment of 2D cell lines, which requires adaptation to monolayer growth on plastic surfaces, tumor organoid cultures are much easier to derive, can be expanded indefinitely ex vivo, and upon xenografting/allografting undergo polyclonal expansion and efficiently produce tumors that preserve the cellular heterogeneity and drug response profiles of the original tumors (AA Duarte, E Gogola, $\mathrm{N}$ Sachs, et al., in prep.). For example, we found the differential olaparib sensitivity of isogenic treatment-naïve and PARPi-resistant KB1P mammary tumors to be stable upon organoid derivation and subsequent retransplantation. Using CRISPR-Cas9 technology, we were able to introduce Trp53bpl frameshift mutations in the treatment-naïve KB1P organoid line and demonstrate that this permutation rendered the organoid-derived tumors refractory to olaparib. We are exploiting the KB1P tumor organoid platform to test additional candidate drug resistance genes for their in vivo relevance, including candidates retrieved from forward genetic screens and from sequencing of drug-resistant tumors (Fig. 4). Moreover,

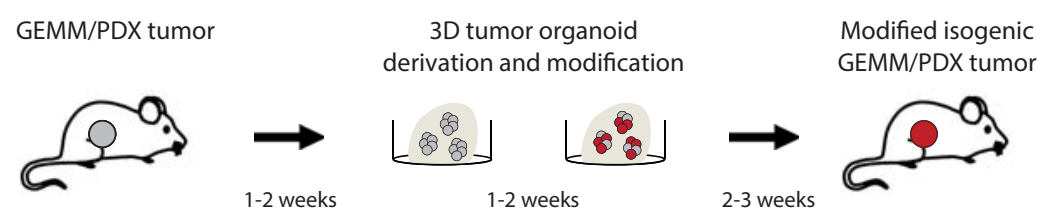

Figure 4. 3D tumor organoid cultures can be rapidly derived from established genetically engineered mouse model (GEMM)/patientderived xenograft (PDX) tumors, modified ex vivo with desired genetic permutations and retransplanted in mice to evaluate in vivo treatment responses. 
given the short latency period and polyclonal tumor outgrowth, GEMM and PDX tumor organoids are particularly amenable for in vivo genetic screens using short-hairpin RNA (shRNA), CRISPR, CRISPRi, and CRISPRa libraries.

\section{CONCLUSION}

A number of known and unknown biological discrepancies inevitably exist between mouse models and humans. Moreover, refinements in mouse modeling should be compliant with practical and ethical issues associated with model establishment. Nevertheless, the systematic and synergistic deployment of complementary in vitro and in vivo platforms (GEMMs, PDX models, organoids, nongermline models) is envisioned to provide a quantum leap in the oncology arena and in breast cancer research in particular. Cutting-edge mouse cancer clinics will enable so-called coclinical trials, in which clinical studies will be paralleled by preclinical intervention studies in mouse avatars. This will allow clinicians to infer in real-time genotype-specific drug response profiles from mouse models and design more effective and long-lasting patient-tailored treatment schemes. The emergence of drug resistance is an invariable and intrinsic consequence of Darwinian tumor growth dynamics, but instead of "whack-a-mole" treatment schedules, coclinical trials could assist in the design of more sophisticated and personalized regimens in which tumors are forced through evolutionary bottlenecks that render them exquisitely sensitive to secondary therapies. Reiteration of this adaptive process is possible only by the use of ever-smarter mouse models, which will ultimately lead to improved long-term management of this devastating disease.

\section{ACKNOWLEDGMENTS}

We are grateful to Peter Bouwman for critical reading of the manuscript. Research in the Jonkers and Rottenberg laboratories described in this review has been supported by grants from the Dutch Cancer Society (NKI 2007-3772, NKI 2008-4116, NKI 2009-4303, NKI 2011-5197, NKI 2011-5220, NKI 2012-5220, NKI 2014-6532, NKI $2015-$ 7877, EMCR 2014-7048), the Netherlands Organization for Scientific Research (NWO), Cancer Genomics Netherlands (CGCNL), Cancer Systems Biology Center (CSBC), NGI Zenith 93512009, VIDI 91711302, VICI 91814643, the Swiss National Science Foundation (310030-156869), the Swiss Cancer Research Foundation (MD-PhD-3446-01-2014), the European Union Seventh Framework Programme (EurocanPlatform project 260791, Infrafrontier-I3 project 312325, CHEMORES project 037665), and the European Research Council (ERC SyG CombatCancer, ERC CoG-681572). Additional support is provided by the Mouse Clinic for Cancer and Aging (MCCA), financed by NWO as part of the National Roadmap for Large-Scale Research Facilities.

\section{REFERENCES}

Amiri-Kordestani L, Basseville A, Kurdziel K, Fojo AT, Bates SE. 2012. Targeting MDR in breast and lung cancer: Discriminating its potential importance from the failure of drug resistance reversal studies. Drug Resist Updat 15: 50-61.

Annunziato S, Kas SM, Nethe M, Yucel H, Del Bravo J, Pritchard C, Bin Ali R, van Gerwen B, Siteur B, Drenth AP, et al. 2016. Modeling invasive lobular breast carcinoma by CRISPR/Cas9-mediated somatic genome editing of the mammary gland. Genes Dev 30: 1470-1480.

Badve S, Dabbs DJ, Schnitt SJ, Baehner FL, Decker T, Eusebi V, Fox SB, Ichihara S, Jacquemier J, Lakhani SR, et al. 2011. Basal-like and triple-negative breast cancers: A critical review with an emphasis on the implications for pathologists and oncologists. Mod Pathol 24: 157-167.

Boelens MC, Nethe M, Klarenbeek S, de Ruiter JR, Schut E, Bonzanni N, Zeeman AL, Wientjens E, van der Burg E, Wessels L, et al. 2016. PTEN loss in E-cadherin-deficient mouse mammary epithelial cells rescues apoptosis and results in development of classical invasive lobular carcinoma. Cell Rep 16: 2087-2101.

Boersma V, Moatti N, Segura-Bayona S, Peuscher MH, van der Torre J, Wevers BA, Orthwein A, Durocher D, Jacobs JJ. 2015. MAD2L2 controls DNA repair at telomeres and DNA breaks by inhibiting $5^{\prime}$ end resection. Nature 521: $537-540$

Borst MJ, Ingold JA. 1993. Metastatic patterns of invasive lobular versus invasive ductal carcinoma of the breast. Surgery 114: 637-641; discussion 641-642.

Boussadia O, Kutsch S, Hierholzer A, Delmas V, Kemler R. 2002. E-cadherin is a survival factor for the lactating mouse mammary gland. Mech Dev 115: 53-62.

Bouwman P, Jonkers J. 2008. Mouse models for BRCA1 associated tumorigenesis: From fundamental insights to preclinical utility. Cell Cycle 7: 2647-2653.

Bouwman P, Jonkers J. 2012. The effects of deregulated DNA damage signalling on cancer chemotherapy response and resistance. Nat Rev Cancer 12: 587-598.

Bouwman P, Aly A, Escandell JM, Pieterse M, Bartkova J, van der Gulden H, Hiddingh S, Thanasoula M, Kulkarni A, Yang $\mathrm{Q}$, et al. 2010. 53BP1 loss rescues BRCA1 deficiency and is associated with triple-negative and BRCA-mutated breast cancers. Nat Struct Mol Biol 17: 688-695.

Bryant HE, Schultz N, Thomas HD, Parker KM, Flower D, Lopez E, Kyle S, Meuth M, Curtin NJ, Helleday T. 2005. Specific killing of BRCA2-deficient tumours with inhibitors of poly(ADP-ribose) polymerase. Nature 434: 913-917.

Bunting SF, Callen E, Wong N, Chen HT, Polato F, Gunn A, Bothmer A, Feldhahn N, Fernandez-Capetillo O, Cao L, et al. 2010. 53BP1 inhibits homologous recombination in Brcaldeficient cells by blocking resection of DNA breaks. Cell 141: $243-254$.

Callen E, Di Virgilio M, Kruhlak MJ, Nieto-Soler M, Wong N, Chen HT, Faryabi RB, Polato F, Santos M, Starnes LM, et al. 2013. 53BP1 mediates productive and mutagenic DNA repair through distinct phosphoprotein interactions. Cell 153: $1266-$ 1280 .

Cancer Genome Atlas Network. 2012. Comprehensive molecular portraits of human breast tumours. Nature 490: 61-70.

Cardoso F, van't Veer LJ, Bogaerts J, Slaets L, Viale G, Delaloge S, Pierga JY, Brain E, Causeret S, DeLorenzi M, et al. 2016. 70-gene signature as an aid to treatment decisions in early-stage breast cancer. $N$ Engl J Med 375: 717-729.

Chapman JR, Barral P, Vannier JB, Borel V, Steger M, TomasLoba A, Sartori AA, Adams IR, Batista FD, Boulton SJ. 2013. RIF1 is essential for 53BP1-dependent nonhomologous end joining and suppression of DNA double-strand break resection. Mol Cell 49: 858-871

Chaudhuri AR, Callen E, Ding X, Gogola E, Duarte AA, Lee JE, Wong N, Lafarga V, Calvo JA, Panzarino NJ, et al. 2016. Replication fork stability confers chemoresistance in BRCA-deficient cells. Nature 535: 382-387. 
Ciriello G, Gatza ML, Beck AH, Wilkerson MD, Rhie SK, Pastore A, Zhang H, McLellan M, Yau C, Kandoth C, et al. 2015. Comprehensive molecular portraits of invasive lobular breast cancer. Cell 163: 506-519.

Clevers H. 2016. Modeling development and disease with organoids. Cell 165: 1586-1597.

Collier LS, Carlson CM, Ravimohan S, Dupuy AJ, Largaespada DA. 2005. Cancer gene discovery in solid tumours using transposon-based somatic mutagenesis in the mouse. Nature 436: $272-276$.

Deeks ED. 2015. Olaparib: First global approval. Drugs 75: 231-240.

Derksen PW, Liu X, Saridin F, van der Gulden H, Zevenhoven J, Evers B, van Beijnum JR, Griffioen AW, Vink J, Krimpenfort $\mathrm{P}$, et al. 2006. Somatic inactivation of E-cadherin and p53 in mice leads to metastatic lobular mammary carcinoma through induction of anoikis resistance and angiogenesis. Cancer Cell 10: $437-449$.

Derksen PW, Braumuller TM, van der Burg E, Hornsveld M, Mesman E, Wesseling J, Krimpenfort P, Jonkers J. 2011. Mammary-specific inactivation of E-cadherin and p53 impairs functional gland development and leads to pleomorphic invasive lobular carcinoma in mice. Dis Model Mech 4: 347 358.

Desmedt C, Zoppoli G, Gundem G, Pruneri G, Larsimont D, Fornili M, Fumagalli D, Brown D, Rothe F, Vincent D, et al. 2016. Genomic characterization of primary invasive lobular breast cancer. J Clin Oncol 34: 1872-1881.

Di Virgilio M, Callen E, Yamane A, Zhang W, Jankovic M, Gitlin AD, Feldhahn N, Resch W, Oliveira TY, Chait BT, et al. 2013. Rifl prevents resection of DNA breaks and promotes immunoglobulin class switching. Science 339: $711-715$.

Drost R, Bouwman P, Rottenberg S, Boon U, Schut E, Klarenbeek S, Klijn C, van der Heijden I, van der Gulden H, Wientjens E, et al. 2011. BRCA1 RING function is essential for tumor suppression but dispensable for therapy resistance. Cancer Cell 20: 797-809.

Drost R, Dhillon KK, van der Gulden H, van der Heijden I, Brandsma I, Cruz C, Chondronasiou D, Castroviejo-Bermejo $\mathrm{M}$, Boon U, Schut E, et al. 2016. BRCA $1^{185 \text { delAG }}$ tumors may acquire therapy resistance through expression of RING-less BRCA1. J Clin Invest 126: 2903-2918.

Droufakou S, Deshmane V, Roylance R, Hanby A, Tomlinson I, Hart IR. 2001. Multiple ways of silencing E-cadherin gene expression in lobular carcinoma of the breast. Int J Cancer 92: 404-408.

Dupuy AJ, Akagi K, Largaespada DA, Copeland NG, Jenkins NA. 2005. Mammalian mutagenesis using a highly mobile somatic Sleeping Beauty transposon system. Nature 436: 221-226.

Escribano-Diaz C, Orthwein A, Fradet-Turcotte A, Xing M, Young JT, Tkac J, Cook MA, Rosebrock AP, Munro M, Canny $\mathrm{MD}$, et al. 2013. A cell cycle-dependent regulatory circuit composed of 53BP1-RIF1 and BRCA1-CtIP controls DNA repair pathway choice. Mol Cell 49: 872-883.

Evers B, Jonkers J. 2006. Mouse models of BRCA1 and BRCA2 deficiency: Past lessons, current understanding and future prospects. Oncogene 25: 5885-5897.

Evers B, Schut E, van der Burg E, Braumuller TM, Egan DA, Holstege H, Edser P, Adams DJ, Wade-Martins R, Bouwman P, et al. 2010. A high-throughput pharmaceutical screen identifies compounds with specific toxicity against BRCA2-deficient tumors. Clin Cancer Res 16: 99-108.

Farmer H, McCabe N, Lord CJ, Tutt AN, Johnson DA, Richardson TB, Santarosa M, Dillon KJ, Hickson I, Knights C, et al. 2005. Targeting the DNA repair defect in BRCA mutant cells as a therapeutic strategy. Nature 434: 917-921.

Fatehullah A, Tan SH, Barker N. 2016. Organoids as an in vitro model of human development and disease. Nat Cell Biol 18: $246-254$.

Hidalgo M, Amant F, Biankin AV, Budinska E, Byrne AT, Caldas C, Clarke RB, de Jong S, Jonkers J, Maelandsmo GM, et al. 2014. Patient-derived xenograft models: An emerging platform for translational cancer research. Cancer Discov 4: 998-1013.

Holstege H, van Beers E, Velds A, Liu X, Joosse SA, Klarenbeek S, Schut E, Kerkhoven R, Klijn CN, Wessels LF, et al. 2010. Cross-species comparison of aCGH data from mouse and human BRCA1- and BRCA2-mutated breast cancers. BMC Cancer 10: 455.

Huijbers IJ, Bin Ali R, Pritchard C, Cozijnsen M, Kwon MC, Proost N, Song JY, de Vries H, Badhai J, Sutherland K, et al. 2014. Rapid target gene validation in complex cancer mouse models using re-derived embryonic stem cells. EMBO Mol Med 6: 212-225.

Jaspers JE, Rottenberg S, Jonkers J. 2009. Therapeutic options for triple-negative breast cancers with defective homologous recombination. Biochim Biophys Acta 1796: 266-280.

Jaspers JE, Kersbergen A, Boon U, Sol W, van Deemter L, Zander SA, Drost R, Wientjens E, Ji J, Aly A, et al. 2013. Loss of 53BP1 causes PARP inhibitor resistance in Brcalmutated mouse mammary tumors. Cancer Discov 3: 68-81.

Jonkers J, Meuwissen R, van der Gulden H, Peterse H, van der Valk M, Berns A. 2001. Synergistic tumor suppressor activity of BRCA2 and p53 in a conditional mouse model for breast cancer. Nat Genet 29: 418-425.

Ledermann J, Harter P, Gourley C, Friedlander M, Vergote I, Rustin G, Scott C, Meier W, Shapira-Frommer R, Safra $\mathrm{T}$, et al. 2012. Olaparib maintenance therapy in platinumsensitive relapsed ovarian cancer. $N$ Engl $J$ Med 366: $1382-1392$.

Ledermann J, Harter P, Gourley C, Friedlander M, Vergote I, Rustin G, Scott CL, Meier W, Shapira-Frommer R, Safra T, et al. 2014. Olaparib maintenance therapy in patients with platinum-sensitive relapsed serous ovarian cancer: A preplanned retrospective analysis of outcomes by BRCA status in a randomised phase 2 trial. Lancet Oncol 15: 852-861.

Liu X, Holstege H, van der Gulden H, Treur-Mulder M, Zevenhoven J, Velds A, Kerkhoven RM, van Vliet MH, Wessels LF, Peterse JL, et al. 2007. Somatic loss of BRCA1 and p53 in mice induces mammary tumors with features of human BRCA1-mutated basal-like breast cancer. Proc Natl Acad Sci 104: 12111-12116

Maddalo D, Manchado E, Concepcion CP, Bonetti C, Vidigal JA, Han YC, Ogrodowski P, Crippa A, Rekhtman N, de Stanchina $\mathrm{E}$, et al. 2014. In vivo engineering of oncogenic chromosomal rearrangements with the CRISPR/Cas9 system. Nature 516: 423-427.

Martinez V, Azzopardi JG. 1979. Invasive lobular carcinoma of the breast: Incidence and variants. Histopathology 3: 467488.

Michaut M, Chin SF, Majewski I, Severson TM, Bismeijer T, de Koning L, Peeters JK, Schouten PC, Rueda OM, Bosma AJ, et al. 2016. Integration of genomic, transcriptomic and proteomic data identifies two biologically distinct subtypes of invasive lobular breast cancer. Sci Rep 6: 18517 .

Moll R, Mitze M, Frixen UH, Birchmeier W. 1993. Differential loss of E-cadherin expression in infiltrating ductal and lobular breast carcinomas. Am J Pathol 143: 1731-1742.

Nik-Zainal S, Davies H, Staaf J, Ramakrishna M, Glodzik D, Zou X, Martincorena I, Alexandrov LB, Martin S, Wedge DC, et al. 2016. Landscape of somatic mutations in 560 breast cancer whole-genome sequences. Nature 534: 47-54.

Oplustil O'Connor L, Rulten SL, Cranston AN, Odedra R, Brown H, Jaspers JE, Jones L, Knights C, Evers B, Ting A, et al. 2016. The PARP inhibitor AZD2461 provides insights into the role of PARP3 inhibition for both synthetic lethality and tolerability with chemotherapy in preclinical models. Cancer Res doi: 10.1158/0008-5472.CAN-15-3240.

Patch AM, Christie EL, Etemadmoghadam D, Garsed DW, George J, Fereday S, Nones K, Cowin P, Alsop K, Bailey $\mathrm{PJ}$, et al. 2015. Whole-genome characterization of chemoresistant ovarian cancer. Nature 521: 489-494.

Perou CM, Sorlie T, Eisen MB, van de Rijn M, Jeffrey SS, Rees CA, Pollack JR, Ross DT, Johnsen H, Akslen LA, et al. 2000. 
Molecular portraits of human breast tumours. Nature 406: 747-752.

Platt RJ, Chen S, Zhou Y, Yim MJ, Swiech L, Kempton HR, Dahlman JE, Parnas O, Eisenhaure TM, Jovanovic M, et al. 2014. CRISPR-Cas9 knockin mice for genome editing and cancer modeling. Cell 159: 440-455.

Rottenberg S, Nygren AO, Pajic M, van Leeuwen FW, van der Heijden I, van de Wetering K, Liu X, de Visser KE, Gilhuijs $\mathrm{KG}$, van Tellingen $\mathrm{O}$, et al. 2007. Selective induction of chemotherapy resistance of mammary tumors in a conditional mouse model for hereditary breast cancer. Proc Natl Acad Sci 104: 12117-12122.

Rottenberg S, Jaspers JE, Kersbergen A, van der Burg E, Nygren AO, Zander SA, Derksen PW, de Bruin M, Zevenhoven J, Lau A, et al. 2008. High sensitivity of BRCA1-deficient mammary tumors to the PARP inhibitor AZD2281 alone and in combination with platinum drugs. Proc Natl Acad Sci 105: 17079-17084.

Sánchez-Rivera FJ, Jacks T. 2015. Applications of the CRISPR-Cas9 system in cancer biology. Nat Rev Cancer 15: $387-395$.

Sander JD, Joung JK. 2014. CRISPR-Cas systems for editing, regulating and targeting genomes. Nat Biotechnol 32: $347-$ 355 .

Schouten PC, Marme F, Aulmann S, Sinn HP, van Essen HF, Ylstra B, Hauptmann M, Schneeweiss A, Linn SC. 2015. Breast cancers with a BRCA1-like DNA copy number profile recur less often than expected after high-dose alkylating chemotherapy. Clin Cancer Res 21: 763-770.

Stephens PJ, Tarpey PS, Davies H, Van Loo P, Greenman C, Wedge DC, Nik-Zainal S, Martin S, Varela I, Bignell GR, et al. 2012. The landscape of cancer genes and mutational processes in breast cancer. Nature 486: 400-404.

Swisher EM, Sakai W, Karlan BY, Wurz K, Urban N, Taniguchi T. 2008. Secondary BRCA1 mutations in BRCA1-mutated ovarian carcinomas with platinum resistance. Cancer Res 68: $2581-2586$.

Tao L, van Bragt MP, Laudadio E, Li Z. 2014. Lineage tracing of mammary epithelial cells using cell-type-specific cre-expressing adenoviruses. Stem Cell Reports 2: 770779.

Ter Brugge P, Kristel P, van der Burg E, Boon U, de Maaker M, Lips E, Mulder L, de Ruiter J, Moutinho C, Gevensleben H, et al. 2016. Mechanisms of therapy resistance in patient-derived xenograft models of BRCA1-deficient breast cancer. $J$ Natl Cancer Inst 108: djw148.
Vaidyanathan A, Sawers L, Gannon AL, Chakravarty P, Scott AL, Bray SE, Ferguson MJ, Smith G. 2016. ABCB1 (MDR1) induction defines a common resistance mechanism in paclitaxel- and olaparib-resistant ovarian cancer cells. Br J Cancer 115: $431-441$.

Vargo-Gogola T, Rosen JM. 2007. Modelling breast cancer: One size does not fit all. Nat Rev Cancer 7: 659-672.

Vollebergh MA, Lips EH, Nederlof PM, Wessels LF, Schmidt MK, van Beers EH, Cornelissen S, Holtkamp M, Froklage FE, de Vries EG, et al. 2011. An aCGH classifier derived from BRCA1-mutated breast cancer and benefit of high-dose platinum-based chemotherapy in HER2-negative breast cancer patients. Ann Oncol 22: 1561-1570.

Vollebergh MA, Jonkers J, Linn SC. 2012. Genomic instability in breast and ovarian cancers: Translation into clinical predictive biomarkers. Cell Mol Life Sci 69: 223-245.

Vollebergh MA, Lips EH, Nederlof PM, Wessels LF, Wesseling J, Vd Vijver MJ, de Vries EG, van Tinteren H, Jonkers J, Hauptmann M, et al. 2014. Genomic patterns resembling $B R C A 1$ - and BRCA2-mutated breast cancers predict benefit of intensified carboplatin-based chemotherapy. Breast Cancer Res 16: R47.

Vos CB, Cleton-Jansen AM, Berx G, de Leeuw WJ, ter Haar NT, van Roy F, Cornelisse CJ, Peterse JL, van de Vijver MJ. 1997. E-cadherin inactivation in lobular carcinoma in situ of the breast: An early event in tumorigenesis. Br J Cancer 76: $1131-1133$.

Wang H, Yang H, Shivalila CS, Dawlaty MM, Cheng AW, Zhang F, Jaenisch R. 2013. One-step generation of mice carrying mutations in multiple genes by CRISPR/Cas-mediated genome engineering. Cell 153: 910-918.

Wang J, Aroumougame A, Lobrich M, Li Y, Chen D, Chen J, Gong Z. 2014. PTIP associates with Artemis to dictate DNA repair pathway choice. Genes Dev 28: 2693-2698.

Wang D, Mou H, Li S, Li Y, Hough S, Tran K, Li J, Yin H, Anderson DG, Sontheimer EJ, et al. 2015. Adenovirus-mediated somatic genome editing of Pten by CRISPR/Cas9 in mouse liver in spite of Cas9-specific immune responses. Hum Gene Ther 26: 432-442.

$\mathrm{Xu} \mathrm{G}$, Chapman JR, Brandsma I, Yuan J, Mistrik M, Bouwman P, Bartkova J, Gogola E, Warmerdam D, Barazas M, et al. 2015. REV7 counteracts DNA double-strand break resection and affects PARP inhibition. Nature 521: 541-544.

Zimmermann M, Lottersberger F, Buonomo SB, Sfeir A, de Lange T. 2013. 53BP1 regulates DSB repair using Rif1 to control $5^{\prime}$ end resection. Science 339: 700-704. 


\section{$\$_{\text {CSH\& }}^{\infty}$ Cold Spring Harbor Symposia SYMPOSIA on Quantitative Biology}

\section{Genetic Dissection of Cancer Development, Therapy Response, and Resistance in Mouse Models of Breast Cancer}

Stefano Annunziato, Marco Barazas, Sven Rottenberg, et al.

Cold Spring Harb Symp Quant Biol 2016 81: 141-150 originally published online November 4, 2016

Access the most recent version at doi:10.1101/sqb.2016.81.030924

References This article cites 72 articles, 16 of which can be accessed free at: http://symposium.cshlp.org/content/81/141.full.html\#ref-list-1

Creative This article is distributed under the terms of the

Commons http://creativecommons.org/licenses/by-nc/4.0/, which permits reuse and License redistribution, except for commercial purposes, provided that the original author and source are credited.

Email Alerting Receive free email alerts when new articles cite this article - sign up in Service the box at the top right corner of the article or click here. 